\title{
Passt das?
}

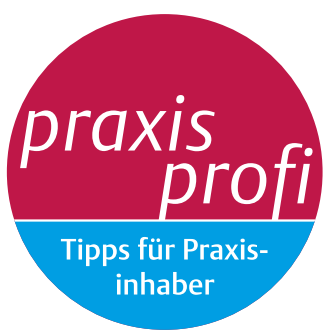

Neu im Team $=$ neues Team Eine neue Mitarbeiterin soll Ihr Team verstärken. Wie sie von den Mitarbeitern aufgenommen wird und wie sich das Team dadurch verändert, hängt von Ihrer Auswahl ab - und davon, wie Sie „die Neue“ präsentieren.
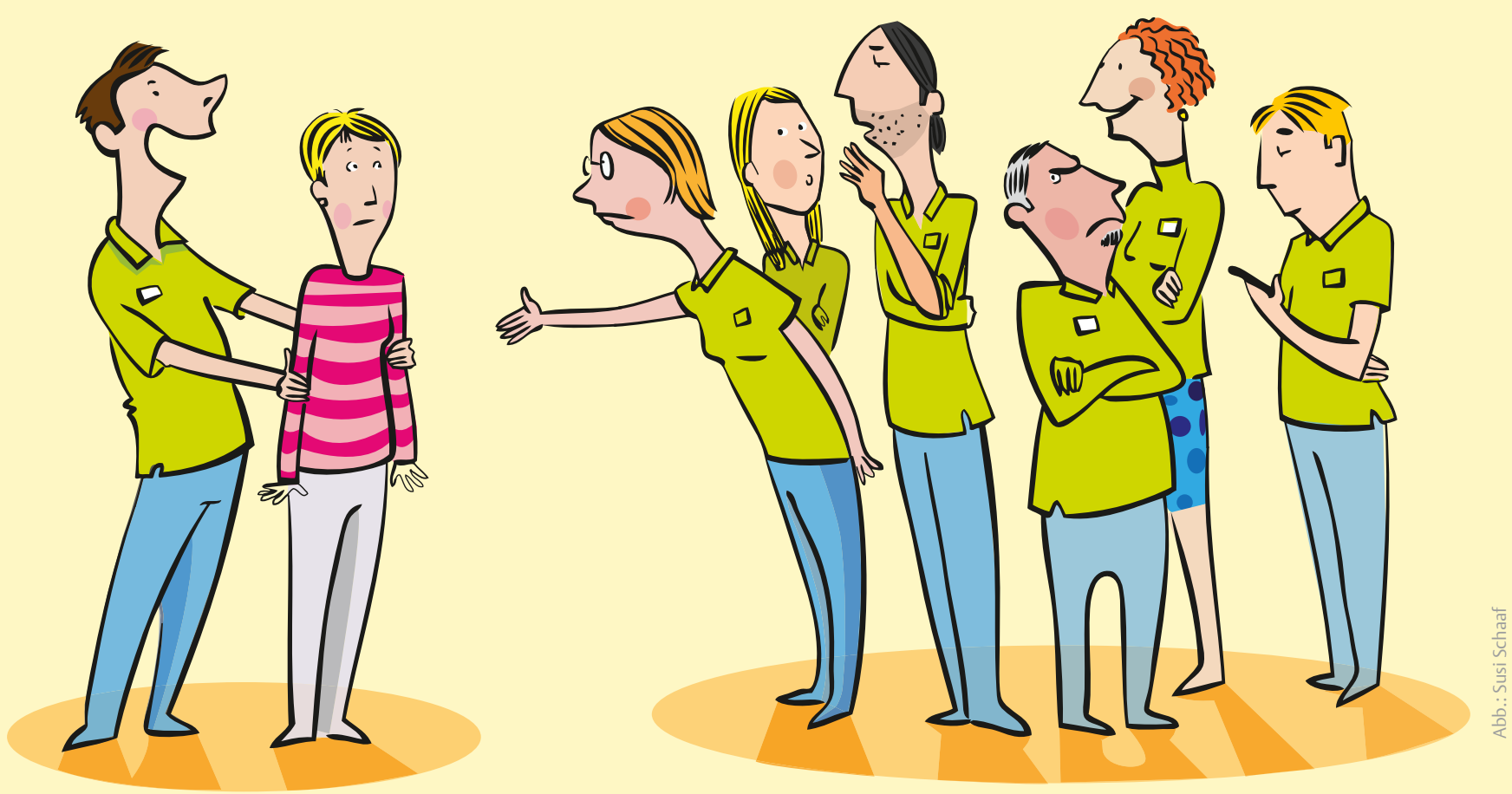

Wie Ihre Mitarbeiter auf „die Neue“ reagieren, haben Sie als Praxisinhaber zu weiten Teilen selbst in der Hand.

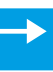

Beim Thema „neue Mitarbeiter“ kreisen fast alle Führungstipps in der Fachliteratur um „die Neue“. Die hohe Bedeutung der ersten par Wochen, eine qualifizierte fachliche Einarbeitung, soziale und kommunikative Integration und bei neu zugezogenen Kollegen auch das „Heimisch-Werden“ mittels Informationen über Infrastruktur der Region sowie adäquate persönliche Betreuung, das alles ist wichtig und will sehr genau bedacht sein. Sie können dabei vieles richtig, aber auch Fehler machen und schlimmstenfalls geschieht die innere Kündigung bereits in den ersten Wochen, wenn die oder der Neue denkt: „Wo bin ich denn hier gelandet? Hier werde ich bestimmt nicht alt!“
Für Sie gilt es einen weiteren Aspekt zu bedenken: Was passiert eigentlich mit Ihrem Team, das nicht nur Neue integrieren, sondern vielleicht auch Aufgaben abgeben oder Änderungen in Abläufen akzeptieren soll? Mehrere grundsätzlich verschiedene Szenarien sind möglich: Sie beteiligen das Team während des Auswahlprozesses und es unterstützt Ihre Entscheidung. Freudige Erwartung wird „der Neuen“ begegnen, die Arbeitsentlastung wird erwartungsvoll herbeigesehnt. Eine ganz andere Situation tritt ein, wenn Sie die
Entscheidung allein treffen. Dann kommt sich das Team im Auswahlprozess übergangen vor oder fremdelt, weil Informationen über „die
95

\section{Einarbeitung ist gelebte} Praxisphilosophie - und eine Form von Marketing
Neue" fehlen. Schwierig wird es auch, wenn Sie „die Neue“ mit Befugnissen ausstatten, die gern jemand aus dem bestehenden Team bekommen hätte. Und noch schwieriger, wenn Sie beispielsweise aus dringendem Personalmangel Zugeständnisse an „die Neue“ machen, die bisher im Team unüblich waren. Das können flexiblere Arbeitszeitregelungen, Urlaubszusagen oder mehr 
Fortbildungstage sein. Ihr Team wird darauf reagieren, jeder auf seine Weise.

Unterschiedlich beeinflusst wird das Team auch je nachdem, ob Sie jemand Erfahrenen, einen Spezialisten oder einen Berufsanfänger einstellen. Es macht ebenfalls einen Unterschied, ob jemand ersetzt wird, der im Team eine echte Lücke hinterlässt, oder aber keinen wirklichen Verlust bedeutet. Und natürlich spielt es eine Rolle, ob der Neueinstellung ein ernsthafter Konflikt vorausging, zum Beispiel eine Kündigung.

Die verschiedenen Faktoren, die zu einer Neueinstellung führen, verlangen von Ihnen bei der Integration der „Neuen“ auch grundverschiedene Vorgehensweisen. Eine standardisierte Einarbeitung wird der Verschiedenheit der Ausgangssituationen ebenso wenig gerecht wie ein immer gleicher Ablauf. Keiner kennt Ihr bestehendes Team so gut wie Sie. Sie kennen den Grund der Neueinstellung und wissen, was „die Neue“ an Können und Erfahrung mitbringt. Sie wissen auch, ob Sie bewusst eine andere Persönlichkeit eingestellt haben, die neuen Schwung ins Team bringen soll, und Sie ahnen auch, wie Ihre Mitarbeiter wohl reagieren werden. Kurzum: Sie tragen anfänglich die alleinige Verantwortung für das Gestalten des Übergangs für alle Beteiligten.

Einarbeitung ist gelebte Praxisphilosophie. Sie präsentieren neuen Teammitgliedern am ersten Tag das, wofür Sie stehen, wie Sie Ihre Mitarbeiter führen und wie Sie Ihre Praxis organisiert haben. Das steigert letztendlich auch Ihre Attraktivität als Arbeitgeber - und ist gutes Marketing.

Ich wünsche Ihnen ein gutes Händchen nicht nur beim Auswählen, sondern auch beim Einarbeiten neuer Mitarbeiter. Praxiserfolg ist planbar! Ihre Anna von Eisenhart Rothe

\section{田 Autorin}

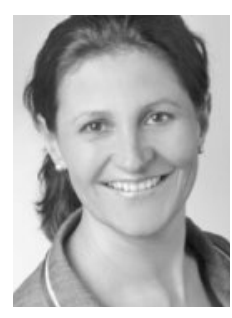

Anna von Eisenhart

Rothe M.A. ist Physiotherapeutin, Diplombetriebswirtin und Wirtschaftsmediatorin. Sie lehrt in diversen Masterstudiengängen Management, Marketing, Personal und Controlling und berät therapeutische Praxen. Außerdem ist sie in den Bereichen Marketingcoaching und Qualitätsmanagement tätig.

\section{Regeln, die Sie beachten sollten}

\section{Sorgen Sie für Transparenz}

Geben Sie allen Beteiligten so viele konstruktive und positive Vorinformationen wie möglich. Für „die Neue“ ist es wichtig zu wissen, ob es Konflikte bezüglich der Vorgänger gab, wie die Alters-, Wissens- und Fortbildungsstruktur im Team ist, ob sie Privilegien hat, ob Vorbehalte gegenüber ihrer Qualifikation bestehen usw. Vermeiden Sie bei der Informationsweitergabe persönliche Details oder emotional belastete alte Geschichten. Kurze knappe Sätze mit reiner Sachinformation reichen aus. Es geht nicht um „lästern“ oder „vorwarnen“, sondern um das Erfassen anspruchsvoller zwischenmenschlicher Situationen.

Für Ihr Team ist es wichtig zu wissen, warum Sie sich für diese Bewerberin entschieden haben. Ihre Auswahlkriterien, die relevanten Eckdaten der „Neuen“ wie Familienstand, Kinderzahl, Fortbildungs-Portfolio, ein kurzer biografischer Abriss, all das interessiert Mitarbeiter an neuen Kollegen. Das Interesse an neuen Kollegen ist in der Regel groß. Einfach direkt fragen mag man den noch fremden Mensch aus Höflichkeit aber nicht. Folglich sind Sie es als Praxisinhaber, der das Informationsbedürfnis aller Beteiligten steuern kann. Ebenso können Sie neue Mitarbeiter mit einem kurzen Abriss über die einzelnen Teammitglieder informieren. Ob Sie das unter vier Augen oder in der ersten gemeinsamen Teambesprechung tun, ist Geschmackssache. Rückfragen werden eher gestellt, wenn weniger Menschen dabei sind.

\section{Formulieren Sie Ihre Erwartungen}

Legen Sie offen, was Sie sich als Praxisinhaber von Ihrer Personalentscheidung versprechen. Sie haben sich bei der Entscheidungsfindung gezielt Gedanken gemacht. Vielleicht erhoffen Sie sich positive Impulse, einen zusätzlichen neuen Praxisschwerpunkt, längere Öffnungszeiten durch mehr Therapeuten oder auch eine Reduktion des sensiblen Themas Therapeutenwechsel. All das müssen alle Beteiligten von Beginn an von Ihnen hören.

Diese Vorgehensweise geht in die Richtung des Führens durch Zielvereinbarung - das sogenannte „Management by Objectives“ (๑ ZUSATZINFO unter www.thieme-connect.de/ products/ergopraxis > „1/17“): Sie geben Ihre Erwartungen vor und besprechen dann mit den Beteiligten, ob diese Ziele realistisch und umsetzbar sind. Stellen Sie die positiven Konsequenzen für das Erreichen Ihrer Ziele für die Mitarbeiter heraus. Übrigens: Dies ist ein perfekter Zeitpunkt für die Weiterentwicklung Ihrer Praxis. Es macht nämlich keinen Sinn, neue Mitarbeiter an das zu gewöhnen, was Sie eigentlich verändern wollen.

\section{Planen Sie gemeinsame Zeit ein}

Menschen brauchen Zeit um sich zu beschnuppern. Sie können das Team ein gemeinsames Willkommens-Frühstück vorbereiten lassen. Das wird auch deshalb gut ankommen, weil es in der Arbeitszeit stattfindet. Sie können nach Arbeitsende alle zusammen essen gehen oder Sie laden Ihre Mitarbeiter beispielsweise zum „Wintergrillen“ ein. Wichtig ist, etwas zusammen zu machen. Geben Sie bewusst Zeit zum Kennenlernen, denn während des Arbeitsalltags fehlt diese Zeit.

Eine neue Stelle anzutreten ist für jeden inhaltlich und zwischenmenschlich mit Stress verbunden. Tragen Sie dieser Tatsache Rechnung. Investieren Sie in gemeinsame Zeit, das ist wichtiger als die eventuell sonst üblichen jährlichen Events wie Betriebsausflug, Weihnachtsfeier oder Geburtstage. 

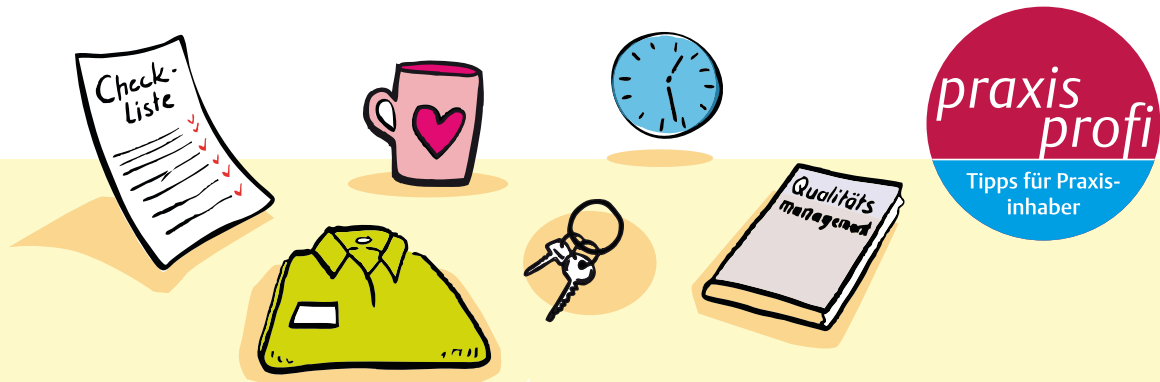

\section{Organisieren Sie die ersten Tage mit Bedacht}

Am ersten Arbeitstag prallt eine Flut von Informationen auf Neue ein. Kanalisieren Sie diese. Im Abstand von fünf Minuten alle Teammitglieder vorzustellen ist zu viel. Wer sollte sich das merken? Wechseln Sie ab. Stellen Sie mal eine Person vor, geben Sie dann beispielsweise Zeit um etwas nachzulesen, schieben Sie Organisatorisches wie die Schlüsselübergabe ein und machen Sie erst danach wieder mit dem nächsten Kollegen bekannt. Vermeiden Sie den Eindruck, die Themen ruckzuck abzuhaken. Lassen Sie Ihr neues Teammitglied spüren, dass sich jeder in der Praxis ausreichend Zeit für den ersten Kontakt nimmt.

Auch Ihr Team braucht dafür Raum. Planen Sie daher in wohldosierten zeitlichen Abständen einen Freitermin ein und was in dieser Zeit geschehen soll. Überlassen Sie die Einführung nicht nur der Rezeptionkraft, auch Ihre therapeutischen Mitarbeiter wollen, sollen und können sich beteiligen. Kombinieren Sie persönliches Kennenlernen mit Informationen über im Team verteilte Aufgaben wie Wäsche, Terminplanung, Werbemaßnahmen, Dienstkleidung, Arbeitszeiten, Regelungen bezüglich der Kaffeekasse, Einrichten der E-Mail-Adresse und Zugangsdaten usw. Der positive Nebeneffekt: Ihr neues Teammitglied kann sich so die Dichte und Fülle an Informationen viel besser merken.

Informieren Sie Ihr Team rechtzeitig, wer wann mit seinem Einsatz an der Reihe ist. Nichts ist peinlicher, als wenn „die Neue“ den Raum betritt und Ihr Mitarbeiter reagiert: „Oh, ich wusste gar nicht, dass Sie zu mir kommen! Toll, dass ich das auch mal erfahre!“ Dann weiß „die Neue“ sofort, dass es mit dem Informationsfluss in Ihrer Praxis nicht zum Besten bestellt ist.

\section{Bieten Sie strukturiert Hilfestellung}

Erstellen Sie beispielsweise eine Checkliste, wer in Ihrer Praxis für welches Thema Spezialist oder Ansprechpartner ist. Planen Sie in den ersten Monaten regelmäßige ca. 15-minütige Check-ups ein, in denen Sie die Einarbeitung weiter begleiten. Der Bedarf ist oft geringer, aber das Angebot, sich Zeit zu nehmen, ist ein wichtiges Signal an neue Mitarbeiter.

Haben Sie ein Qualitätsmanagement-Handbuch? Wenn ja, ist jetzt der richtige Zeitpunkt, um zu überprüfen, ob darin klar und deutlich steht, was für Neue relevant ist. Checken Sie das Handbuch vorher nochmals auf Vollständigkeit. Sind Kompetenzen und Verantwortungsbereiche aller Teammitglieder bzw. Aufgabenbereiche klar definiert, alle Prozesse erfasst? Wurden Veränderungen schriftlich aktualisiert? Nehmen Sie das Feedback bzw. die Fragen der „Neuen“ als Rückmeldung, ob das Instrument begleitend zur Einarbeitung greift.

\section{Lassen Sie es langsam angehen}

Häufig wird das neue Teammitglied sehnlichst erwartet. Womöglich haben sich eine Menge Überstunden des Teams angehäuft. Nehmen Sie dennoch das Tempo raus. Je mehr „die Neue “ mit Arbeit überschüttet wird, umso eher geschehen Fehler und Missverständnisse. Bitten Sie Ihr Team, Verständnis dafür zu haben, dass es am Anfang sinnvoll ist, es langsam angehen zu lassen. Der Abbau der Überstunden sollte dennoch präzisiert werden. Alle sollten wissen, ab wann das neue Teammitglied voll einsatzfähig sein wird und ab wann überbelastete Kollegen etwas abgeben können. Zudem könnten Sie einen Überstundenabbauplan besprechen. Wenn Ihr Team weiß, dass beispielsweise zwei Monate nach Arbeitsbeginn des neuen Mitarbeiters jeder mal einen Tag pro Woche zum Überstundenabbau frei haben wird, motiviert das, und der Nutzen der Verstärkung im Team wird für alle spürbar. 